\title{
15
}

\section{Dealing with standard components for knowledge intensive CAD}

\author{
S.J.Culley, G.Theobald \\ School of Mechanical Engineering \\ University of Bath \\ Bath, BA27AY, UK \\ 441225826456 (Voice)/826928(Fax) \\ S.J.Culley@bath.ac.uk
}

\begin{abstract}
The design of engineering sub-assemblies is an essential and important part of any machine or system design activity. These sub-assemblies are a combination of custom designed elements and standard components from manufacturers catalogues. This paper describes the way in which these elements can considered in an integrated manner at an early stage in the design process. This is achieved by the development of an integrated hierarchical structure and the parametric representation of catalogue information.
\end{abstract}

\section{Keywords}

Functional modelling, standard components, conceptual design, parametric design, power transmission systems

\subsection{INTRODUCTION}

Engineering products are a combination of systems and sub systems. The function of a product is defined as the conversion between the inputs and the outputs of the system and its sub-systems [Ullman 1993]. The function of a product describes what must be achieved by the design, as opposed to the behaviour which describes how the design performs, although the two are often used interchangeably by engineers[Finger and Rinderle,1989] At the heart of any system are the multiplicity of individual components that dictate the performance over the product life cycle. The authors have identified three types of components, namely bespoke(custom) designed components, standard-designed components and standard-selected components, which will be described in more detail later in the paper. It is thus essential in any knowledge intensive CAD system or functional modelling system that the characteristics of individual components are considered. This paper will describe an approach and architecture that enables components and their characteristics to be dealt with. 


\subsection{STANDARD COMPONENTS}

Most technical products designed in industry are constructed of a complex hierarchy of assemblies, sub-assemblies and components. The definitions of these terms, and the extent to which they encompass elements of the product, are held largely in the minds of individual designers and are therefore vague and open to interpretation. For example, a vehicle designer might consider a car to be an assembly, an axle unit as a sub-assembly, and the wheel bearing as a single component within this hierarchy. The bearing designer, however, would consider the wheel bearing to be an assembly, the caged elements as a sub-assembly and the inner/outer rings, cage and the rolling elements as the components of this assembly.

In an attempt to rationalise the elements of a product during its design, Hubka[1992] considers that a product is a technical system which interacts with other systems and the environment by inputs and outputs to the system. To illustrate this, the product shown in Figure 1,[Pahl and Beitz,1984] is defined by the system boundary S. In addition, further technical systems S1-S5 represent sub-systems of the product at any particular level of abstraction. Increasing division of a system in this way enables the product to be considered at increasing levels of detail, until sub-systems are reached which are commonly found in existing machine systems e.g. screws, bearings, shafts, levers, wheels etc. These are termed "machine elements" by Hubka, or in this paper components.

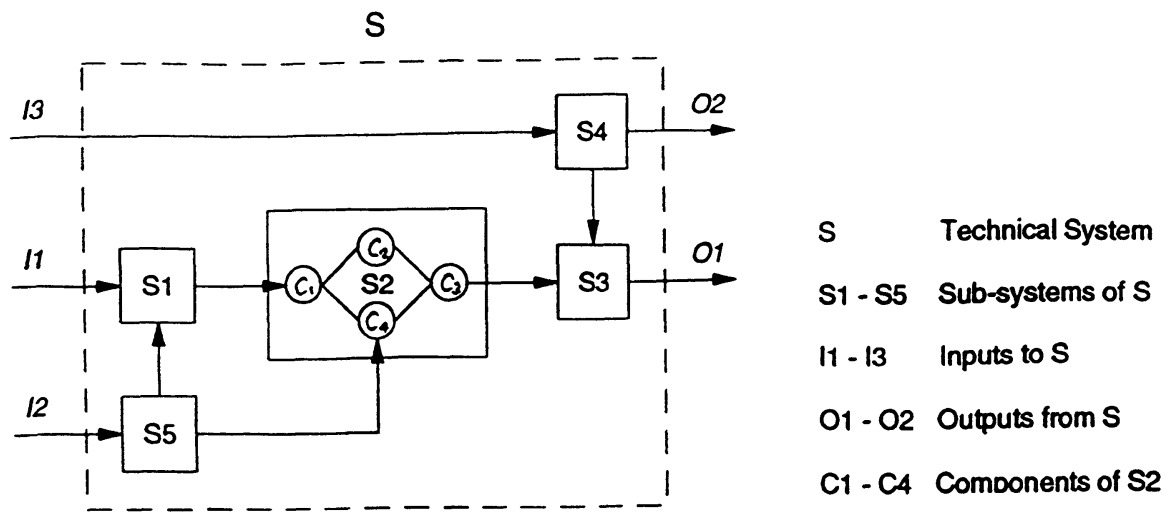

Figure 1. Elements of a Technical System(Pahl and Beitz, 1984) 
This framework does not completely overcome the ambiguities identified earlier, but does provide definitions which can be usefully be used namely:

-A system is a device which converts a set of inputs into a desired set of outputs to carry out a specific function.

-A component is the lowest level of sub-system which is part of a larger system at a particular level of refinement of the design.

Despite their eagerness to advocate their use, most texts and research works do not provide much explanation of what is meant by the term standard components. It is generally considered that they are components purchased from external manufacturers as "bought-out finished" components, or purchased "off-the-shelf" from suppliers[Reinemuth and Birkhofer,1993]

Until now the term standard components has been used to describe a wide range of components, obtained from a variety of sources and distinguished by many different characteristics. This section will address the lack of accuracy in this definition by proposing a classification of engineering components into which standard components can be better placed.

It is proposed that engineering components can be classified according to three primary types.

1. Bespoke-Designed Components

2. Standard-Designed Components

3. Standard-Selected Components

And 4 secondary types which overlap between these as shown in Figure 2

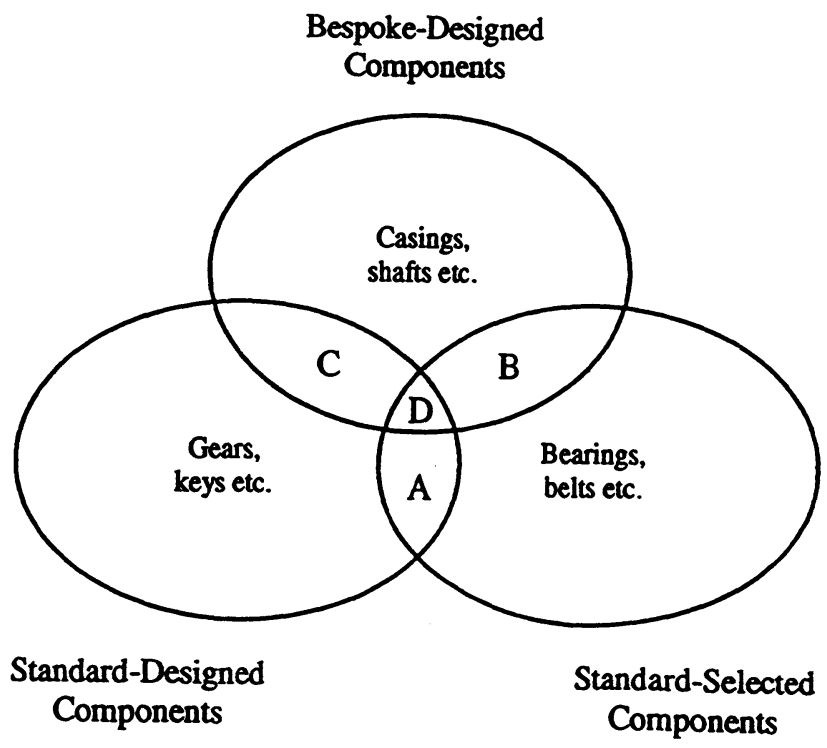

Figure 2 A classification of engineering components 


\subsection{Bespoke-Designed Components}

If components are not standard, then they are bespoke or custom made. Bespoke-designed components are a very wide-range of components which are designed and manufactured to satisfy the needs and requirements of unique problems. Other work[Blessing ,1994] has shown that as many as $40 \%$ of components in a product may be bespoke and therefore cover a wide range of types of component. For each component, there is an infinite range of possible solutions as, unlike standard components, they are not constrained to conform to a set of discrete sizes, except for those imposed by attached standard components. The choice of these attaching standard components may therefore have a great influence on the final design of large portions of the product and its cost.

Since they are designed for unique problems, definitive standards and procedures for their design may not exist, and they may therefore require a considerable amount of creative design and engineering effort to arrive at a possible solution. Typically the design of these components may be based on the knowledge and experience built-up by the designer or gained from similar previous products. During their design, the unique form of the component may be brought about by the complex, multi-function and highly-coupled roles that it will perform. Due to these levels of complexity, their design may well be carried out using 2D and 3D CAD geometric modelling techniques, and analysed using finite element analysis techniques.

\subsection{Standard-Selected Components}

Standard-Selected components are the type of standard component which the designer would normally obtain as "bought-out-finished" from a third-party manufacturer. This type of component requires very little creative or intuitive "design" work since they are selected according to the selection procedures contained in the supplier's catalogues. Since these procedures are defined by individual manufacturers (who may try to preserve commercial secrecy) it may be only the selection procedures, and not the fundamental principles on which they are based, which are published and made available to the designers. The Author has researched the Computer Aided Selection Of Components(CASOC) for a number of years[Culley and Webber 1992, Vogwell and Culley 1991, Wood 1994] and shown the various problems for the developer and benefits for the engineering designer associated with this type of technology

Nevertheless it is very difficult to establish analytical models for the design of these components since these are contained within the selection procedures in the component catalogues. Although this is not a problem to the designer who merely selects components from the catalogue it does present a difficulty to any system that attempts to make this knowledge accessible to the designer in a different way. 


\subsection{Standard-Designed Components}

An additional class of standard components has been identified which are neither bespokedesigned nor standard-selected. Standard-designed components are standard components which are not generally selected from manufacturer specific catalogues. The design and selection of these components is based on well established fundamental principles and equations which have themselves become standardised design procedures. The design standards which describe the design of these components can be recognised at many levels, typically:

- National and international standards e.g. British Standards (BS), International Standards Organisation (ISO), DIN and ASTM etc.

-Industry based standards e.g. Civil Aviation Authority (CAA), Ministry of Defence (MOD) etc.

-Company based standards e.g. National Coal Board (NCB) and Rover Engineering Standards (RES) etc.

An important characteristic of this type of standard component is that the models of the design procedures are not contained within a component catalogue but are well established and widely recognised within the engineering community. Therefore these may be published elsewhere in design handbooks etc. These standards may contain similar types of procedures as those in the suppliers catalogues, i.e. analytical equations, tables, factors and graphs. In addition, some may also contain information about preferred sizes, and procedures which define how this range of components may be extended whilst maintaining conformance to the "standard". This allows a degree of freedom for designers to design and specify standarddesigned components, outside of the normal range, for particular tasks.

\subsection{Secondary Type Components}

This classification is not rigidly defined by these three primary types of components. Figure 2 indicates that their is potential overlap between these component types, and it has been recognised that many components display characteristics of two or possibly all three of these categories. Four types of secondary components have been identified.

Class $A$ components display characteristics of the standard-designed and standard- selected components. These components may be designed to the relevant standards and then selected without further analysis from a range of suppliers components which are defined by size and form only. Typically this may occur with some of the more common types of gears, such as spur gears, which can be purchased "off-the-shelf" from suppliers once they have been designed.

Class $B$ components display characteristics of the standard-selected and bespoke- designed components. These components may be initially selected from suppliers catalogues in the conventional manner and subsequently adapted or modified to the suit the form or function requirements of a particular problem. In this instance the component may require further specialist analysis to ensure correct function. Typically this may occur with pipework which 
must be bent or modified and electric motors which require modified flange mountings to suit the product.

Class $C$ components display characteristics of the standard-designed and bespoke- designed components. These components may be designed as bespoke-designed components but some elements of it are constrained by standardised design practices. Typically this may occur in the design of a bespoke-designed shaft which must adhere to the constraints of a standarddesigned key-way slot.

Class $D$ components display characteristics of all types of components. Typically this may occur in the design of a particular gear. This may be designed as a standard-designed component, selected as an "off-the-shelf" component from a supplier and with a machined hub to suit the attachment method of an existing bespoke shaft.

\subsection{FUNCTIONAL MODELLING}

Functional modelling is used to represent the functional intent of the product as opposed to the geometric intent of the product as performed by the first and second generation CAD systems. To achieve this, it is considered that functional modelling can be approached in one of two ways [Johnson and Thornton 1991]

- Quantitatively by considering the mathematical models which link the form of the component to its function.

- Qualitatively by a more philosophical approach which links the form of the component to a grammatical description of the required function of the component.

In both cases, input to the functional model is the required function that the system must perform. In the first this may be a numerical description of the required function, e.g. the value of the power to be transmitted by a shaft, and in the second a noun-verb description, e.g. the function of a bearing is to hold shaft. As Johnson identifies, the second will be the only way to design truly original solutions however, despite a number of attempts, the formalisation of such a grammar is proving to be very difficult for all but the narrowest of domains [Chakrabarti 1991].

Since functional modelling has the potential to cover a very wide range of tasks and activities in the design process, many researchers have proposed structured approaches for both their own programmes and for the wider research community [Duffy and Dixon, 1990,Ullman,1992a] to enable more effective research into these types of tools. From these, a common consensus has largely been reached which is typified by the taxonomy proposed by Finger \& Dixon [1989]. This identifies three basic types of design problem and hence three general classes of computer-based design tools.

- Parametric design tools

- Configuration design tools

- Conceptual design tools.

Many functional modelling tools have been researched and developed within this taxonomy and there is an extensive range of research in these areas. This paper is concerned with the use 
of standard components in functional modelling and one example of this type of work will be reviewed.

\subsection{Functional Modelling and Standard components}

The parametric design of simple systems with standard components is reported by Ward \& Seering [1987]. This describes a system that allows a simple shaft assembly to be represented in the computer. By a process of message passing, between the components, and design calculations a parametric description of the complete assembly can be made, thus allowing its evaluation. Like other systems, the complex process of message passing requires that the problem is pre-defined in the computer and prevents the system from being used easily for a wide range of problems.

This problem was recognised by the authors and, in an extension to this work, has been partly overcome in the development of the design compiler [Ward and Seering, 1990]. This system allows the designer to specify the structure of the design in the computer at the outset of the problem.

This is a good attempt to incorporate standard catalogue components during the design process, however, it is limited in a number of ways. Firstly, in order to eliminate the catalogue components they must be stored in the computer in a rigid hierarchical structure. This prescribes the way in which all manufacturers should organise their product catalogues in the future, and does not utilise the CASOC type software which are currently available and being developed. And secondly, potential catalogue components are eliminated by excluding those which fall outside of the desired parameter range. This requires that all elimination can be carried out directly on the data in the catalogue. This therefore limits the potential of the approach taken by the design compiler and another must therefore be sought, which is described later in this paper.

Some of the issues mentioned above, regarding the insufficient use of standard components, were addressed by Corderoy et al [1991] who propose a system for the conceptual design of engineering assemblies. The concept of Computer Integrated Design (CID), as it is known, is based on observations about the nature of common mechanical engineering assemblies. This recognises that an assembly is constructed of a configuration of many sub-assemblies and individual components. To manage the complex interactions of these configurations within the complete assembly a hierarchy of control modules is used, shown in Figure 3, which each act as a database for the design of a configuration level within the assembly. It is the left hand 'leg' of this that the system described in the next section has been developed to address.

In general, the task of computer-based conceptual design involves the synthesis of functional solutions from a functional description of the problem. Since the outcome of this type of tool is a function structure suitable for embodiment, it will not involve the use of standard components. Any utilisation of standard components will therefore be carried out, at the earliest, in the configuration design tools. 


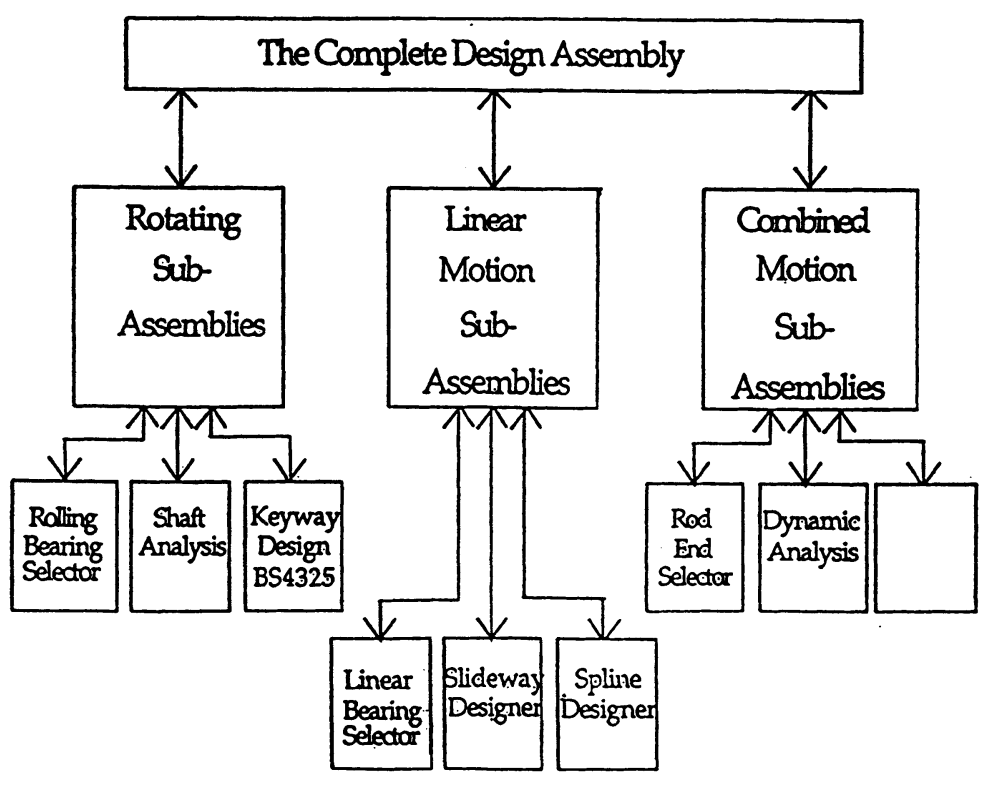

Figure 3 The concept of computer integrated design

\subsection{MODELLING WITH STANDARD COMPONENTS}

It is clear from the above sections that standard components are an essential element in any system and have largely been overlooked in the work on functional modelling. It clearly an area of knowledge where engineers need additional support so that they are able to make informed decisions about the long term operation of their systems and sub-systems. To address these issues the Authors have worked on a systems known as the Bath University Design System(BUDS) which has been conceived to address the following issues:-

- The conceptual design and modelling of engineering assemblies.

- The incorporation of standard components in the early stages of the design process.

- The optimisation of engineering assemblies of standard components.

The philosophy behind the system is to enable the engineering designer to rapidly evaluate and develop a concept based on real components, analytical routines and engineering standards in an integrated manner, see figure 4 . Thus once the concept has been entered 
into the system and the various operations undertaken the designer would know that prime analytical constraints are not violated, standards are satisfied and components that will support the loads or transmit the power,etc. are available from manufacturers. The designer is then able to optimise the concept before moving into the more detailed embodiment stages of the design. To support this aim the system has a number of key attributes as follows:

- Be a user-led aide which will allow designers to make design decisions and manipulate the system to their own requirements.

- Allow data to be entered, used and modified in a common format.

- Specify a minimum amount of data to define a design problem.

- Present a graphical representation of the problem or solution to the designer.

- Provide appropriate help facilities and intelligent feedback to the designer.

\subsection{The Bath University Design System (BUDS) -Overview}

BUDS has been developed as a computer-based support tool for the adaptive design of engineering systems at the conceptual and embodiment stages of design. An overview of the system is shown in Figure 4. Once this concept variant has been represented in the system, a range of tools enable this concept to be: Designed, analysed, evaluated and refined for functional suitability using the Parametric Design Algorithms, optimised using the General Purpose Optimisation System, Embodied with suitable standard components using the Detail Specification Tools.

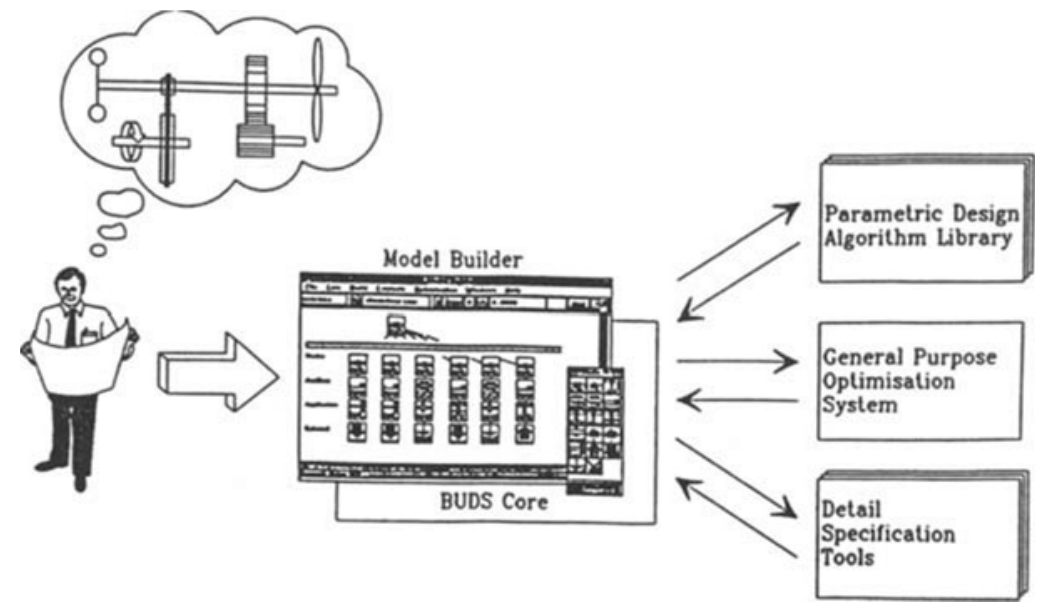

Figure 4. Overview of the Bath University Design system (BUDS) 
This process progresses an initial concept variant to a part-embodied design solution. The result of these phases is a functionally and dimensionally optimised conceptual layout of the design. Standard components are considered in two ways as follows:-

\section{First-stage - Parametric Design Algorithms}

During the conceptual design phase a Parametric Design Algorithm (PDA) is used to carry out the preliminary design, analysis and evaluation of the functional suitability for all sub-systems, components and features in the concept. This is done to ensure that the complete system is able to fulfil the overall function defined by the designer.

\section{Second-stage - Detail Specification Tools}

The second-stage for incorporating standard components is by their detailed selection and specification using Detail Specification Tools (DSTs). These are third-party CASOC-type component selection packages which are used to select real components from the output of the PDAs.

\subsubsection{Design Optimisation}

It has been proposed [Theobald et al.,1993] that the optimisation of a design model that is developed using BUDS is carried out using a three-stage strategy which is shown in Figure 5. These are summarised here for completeness but are outside the intended scope of this paper.

\section{First-stage - Configuration Optimisation}

This is carried out to develop the optimum function structure and configuration of components to satisfy the overall function of the system. At present this is a manual process carried out by the designer who decides the layout of the concept and the types of component which satisfy the solution principles

\section{Second-stage - Parametric Optimisation}

Once a suitable configuration of the concept has been established, this process is used to optimise the variable functional and dimensional parameters of the overall system and its components. This is done before the detailed selection of the standard components using the Detail Specification Tools is carried out.

\section{Third-stage - Relational Optimisation}

After selecting and specifying a range of suitable components using the Detail Specification Tools, this process is used to optimise the combination of these. 


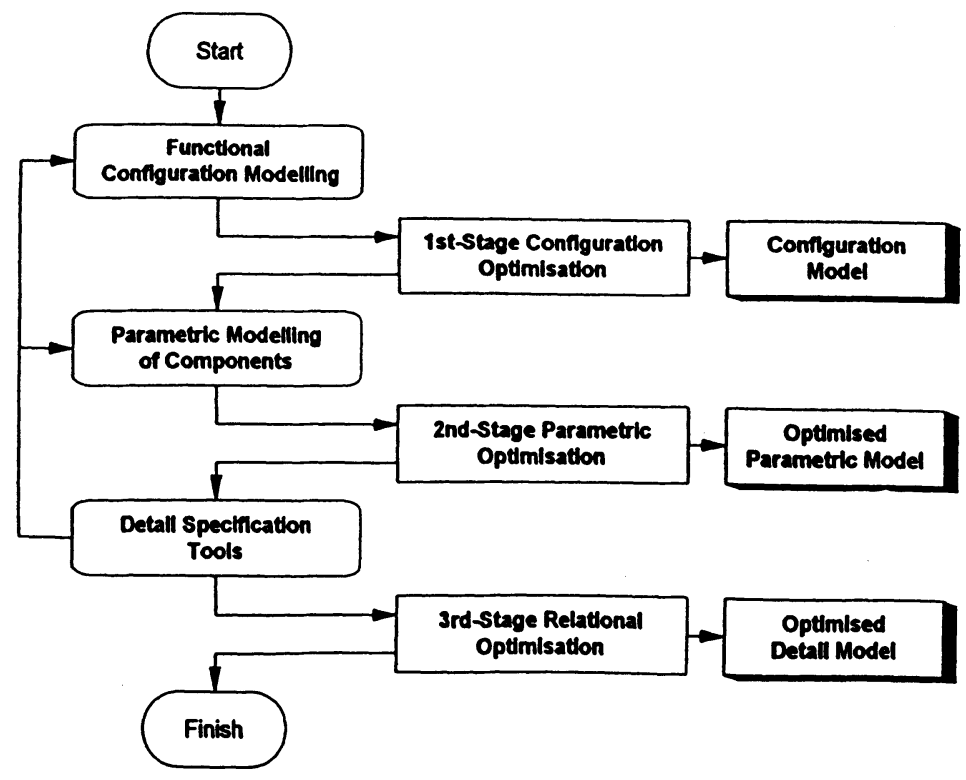

Figure 5 Stages of optimisation.

\subsection{The Architecture of BUDS}

The system architecture of BUDS is shown in Figure 6. It consists of 5 main software modules.

- The Model Builder - This is the main user interface for the system, where the designer is able to represent the concept model of the design using the icons in the toolbox, and can initiate control of the system.

- BudsCore - This is the main control system for BUDS. It is also the main database which stores the concept design model using an object-oriented approach. This is described in more detail in the next Section.

- The Parametric Design Algorithm Library (PEDAL) - This is contains the Parametric Design Algorithms for each type of component that can be used in the modelling system.

- The General Purpose Optimisation System (GPOS) - This is an independent optimisation system which can be linked to BUDS when optimisation is required.

- The Detail Specification Tools (DST's) - These are a range of third-party software packages which are used for the detailed selection and specification of standard components. 


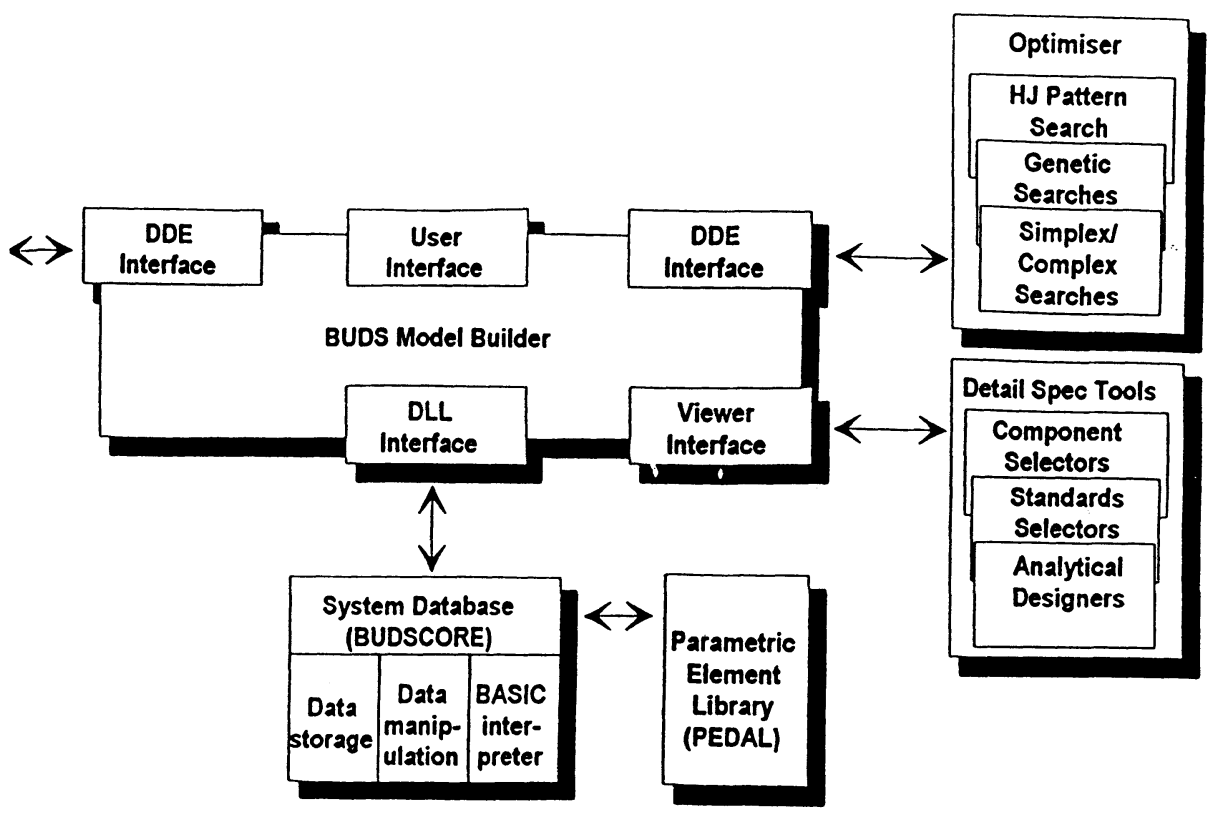

\section{Figure 6 Architecture of BUDS}

\subsection{Parametric Design Algorithms (PDA) for standard components}

It is suggested in models of the design process [Pahl and Beitz,1984, Ullman,1992b] that during the early development of a conceptual design, the designer carries out a number of tasks to evaluate the initial suitability and feasibility of a proposed solution. These decisions are generally based on quick and rough design calculations based on well known and widely used principles. These are often used to determine the major functionally important parameters which are used to evaluate the design. For example, in the conceptual design of an engine con-rod the designer may make rough calculations to determine the buckling limit of the connecting web based on approximate known dimensions. This will be done to evaluate the feasibility of the functional parameters of the engine such as the maximum power and torque output.

This activity is carried out in the BUDS design process using the Parametric Design Algorithms (PDAs). These are essentially software-based representations of the conceptual model for each type of component within the design domain. A PDA is required for all types of engineering components, namely bespoke-designed, standard-selected and standarddesigned components. It is proposed that it is at this stage when standard components should first be considered as elements of the BUDS design model.

It was suggested by Hoover et al.[1991] that an engineering model is used for two purposes: 
-To enable the design to be refined to a more complete state.

-As a means of predicting the behaviour of the system.

These are two of the tasks that are carried out by the PDA. In the PDA, these are referred to as the design and analysis functions respectively. In addition, the PDA is also required to carry out a third function. This is to evaluate the feasibility and suitability of the component to meet its required function. It is important to differentiate between these tasks of design, analysis and evaluation in the PDA.

\subsubsection{PDA Design}

This is the task of estimating and proposing the form, dimensions and other physical attributes of a component to fulfil the functional requirements, and hence refine the component from an abstract state to a more defined one. This information is required for three reasons:

- It enables a minimum amount of information to be proposed and entered about a design during the conceptual stage.

- It creates information which may be necessary for the subsequent analysis and evaluation tasks of the PDA.

- It creates information which is necessary for the subsequent optimisation and detailed specification of components .

\subsubsection{PDA Analysis}

This is the task of examining the functional behaviour of the component based on its physical description. Obviously, analysis of the component cannot be carried out until some element of design has been carried out to determine its physical description, whether speculatively by the designer, by the design element of the PDA, or by constraints imposed by surrounding components of the design model.

\subsubsection{PDA Evaluation}

This is the procedure for making reasoned decisions, based on the form and functional attributes determined above, about the suitability of the component for the task. These will be based on the constraints applied by the PDA, the other components within the system and the requirements specified by the designer. In the case of unsuitability, feedback should be provided to the designer in two ways. By setting the appropriate traffic light of the component in the design model, and advising the designer on the nature of the constraint violation and suggesting ways of how this conflict can be resolved.

\subsection{The Resolve Process}

The Resolve Process is the way in which the system uses the PDAs for the design of each component in a BUDS model. The resolve process is initiated by the designer once the concept design has been modelled and the requirements and constraints about the model have been defined. The way in which this process is managed must address the following issues.

-How do the requirements and constraints get propagated through the model? 
-How are conflicts between different constraints managed ?

-How is it ensured that each component PDA is executed properly?

The outcome of the investigation is a process which manages these addresses these issues. This is referred to as the Resolve Process which consists of three main tasks: Data Propagation, Data Arbitration and PDA Execution.

\subsubsection{Data Propagation}

The data propagation process ensures that the requirements and constraints defined by the designer, optimisation system and the PDAs of the components are propagated to all other components of the model that require them. This process considers each component of the model in turn and, using a variation of the blackboard principle[Engelmore and Morgan, 1988] collates and redistributes the required component parameters. To ensure propagation throughout the complete BUDS model a three-step operation is used.

- Step 1 - The data is propagated up and down the vertical component "chains".

- Step 2 - The data is propagated across the horizontal "nodal" components.

- Step 3 - The data is re-propagated up and down the vertical component "chains".

Information about which component parameters are to be propagated are defined in the generic class definition for the component object. This defines which parameters are to be input and which parameters are to be output for each class of component. For example, in a shaft key component object, the shaft diameter, hub length, power transmitted and shaft speed are input and key cross section, key length and key mass/cost are output for each instance of a shaft key.

\subsubsection{Data Arbitration}

Data arbitration is required to resolve the conflicts encountered during data propagation. This is necessary to ensure that less important requirements and constraints on the system (e.g. default values) do not overwrite more important ones (e.g. values entered by the designer). During data arbitration the following hierarchy of constraints is used:

- Designer entered parameter.

- Optimised parameter.

- PDA calculated parameter.

- Default parameter.

During data propagation, if the blackboard encounters two conflicting parameters the arbitration process uses this order to ensure that the more important value is maintained by either overwriting, or not propagating, a less important parameter. If two equal status parameters are encountered the conflict is brought to the attention of the designer to resolve.

\subsubsection{PDA Execution}

After the data has been propagated and arbitrated for the complete model, the PDA for each component is executed. This is only carried out if the attribute parameters have been changed after the data propagation process and consequently the traffic light is in the intermediate amber state. 
The complete Resolve Process requires the propagate-arbitrate-execute cycle to be carried out twice. This ensures that the components parameters calculated during the first pass are used as constraints for the other components in the second pass. At the end of this second pass the feasibility of the model can be assessed from the status of the traffic lights on each component; if all traffic lights are green then the model is feasible.

\subsection{The Detail Specification Tools (DST)}

The second-stage of the strategy for incorporating standard components is using the Detail Specification Tools (DSTs). These are third-party software applications which are used for the detail design, analysis or catalogue selection of the components.

The detailed design of the components can be carried out after a feasible parametric model is obtained regardless of whether the design has been parametrically optimised or not.

\subsection{MODELLING POWER TRANSMISSION SYSTEMS}

The previous Section has described the generic BUDS system. It was required that the system should be used for the design of a wide range of engineering systems, and the construction of the system allows for this. However, for the implementation of a prototype system it has been configured for the design of rotating power-transmission systems. This is particularly relevant as items such as bearings, gears and belts have a lot of attributes associated with them and have key life equations for example.

\subsection{The Design of Power-Transmission Systems}

The development of computer-based tools for the design of rotating power- transmission systems has been the subject of other research works [Claypole, 1993, Howe et al.1986]. However, these are limited in their ability to model the systems in a flexible and effective way. Two major problems have been identified. Firstly, there is a lack of flexibility in the configuration of a design problem. For these systems, the configuration is effectively hardcoded into the modelling system. Attempting to explore alternative concept configurations quickly and easily would require extensive re-writing of the system. Secondly, in many systems small yet important elements of a power-transmission system are neglected, such as elements which connect components onto the shaft, and connections with the external influences of the system. It is perceived that this is because of a lack of structure to the configurations. It is these important aspects that need to be addressed for more knowledge intensive support for engineering designers.

\subsection{Power-Transmission Component Hierarchy}

Observation of the power-transmission domain reveals a structure which is common to many systems. A more complete structure consists of five elements described below.

- Global element - This defines the engineering domain which is being considered. In this case, it is a power transmission system domain. This element should contain the global attributes and function of the system. 
- Nodal element - This element defines the structure of the global system and how it is decomposed into the configuration of elements. In this case, it describes the basic construction of the shaft into shaft nodes.

- Auxiliary element - This element characterises the connection between the application elements and the structure of the system, i.e. the nodal elements.

- Application element - This element is the primary functional element of the system. In this case, it is the components which transmit power, resist loads etc.

- External element - This element is the interface between the system and the environment in which the system is contained.

\subsubsection{The BUDS Power-Transmission Domain}

For the purposes of this research the prototype system has been populated with the following power-transmission components, features and sub-systems:

- Global element - shaft

- Nodal element - shaft node

- Auxiliary elements - shaft key and free-fit

- Application elements - generic bearing, deep groove bearing, angular contact bearing, generic power-transmission element, gear-pair sub-system, tooth-belt sub-system and an inertial load.

- External elements - input, output and bearing mountings.

Parametric Design Algorithms have been developed for each of these classes of components.

\subsection{Power Transmission Example - The Oil Burner Shaft}

The example in Figure 7 demonstrates how the concept for an oil burner shaft can be modelled by decomposing the problem into a six-node configuration. These are represented by shaft nodes and the following components: the oil atomiser and fan as rotating loads which consume power, the two bearings which are attached to the shaft node by a free-fit and are mounted to the surroundings, the keyed-on gear which drives the oil pump, and the keyed-on toothed-belt which transmits power to the system from the electric motor.

This example illustrates that in some situations the component hierarchy is not completely satisfactory since the oil atomiser, which is manufactured as an integral part of the shaft, does not require an auxiliary component to attach it to the shaft. In this case, the component is left as a null component from the initial template. 


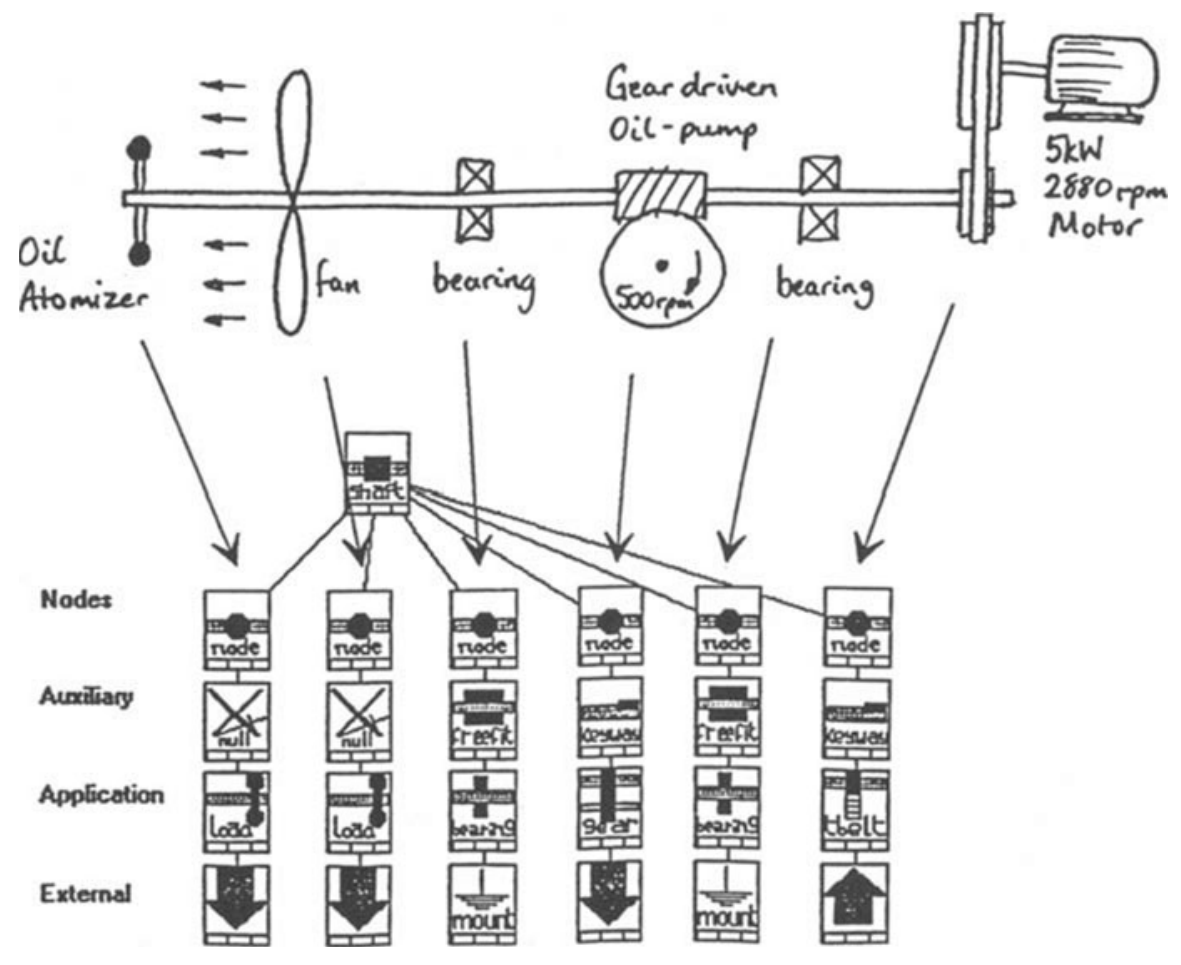

Figure 7 Power Transmission Example- An oil burner shaft.

\subsection{CONCLUSIONS}

It is clear that standard components of whatever description are essential ingredients of any engineering system. their choice and design will have profound implications for the long term performance of any machine or device. It is therefore essential that they are considered as earlier as possible in the design process and are considered in conjunction with ethe rest of the system and not in isolation.

Thus this paper describes a strategy for incorporating standard components into a computer aided design system, which has been implemented. This has been achieved using a two-stage approach. The Parametric Design Algorithms are used for the initial functional modelling and evaluation, and the Detail Specification Tools are used for the final selection of actual components. The system allows the designer to input a minimum amount of data which is then propagated through the system. It also allows constraints and conflicts to be addressed and a 
preliminary viable functional model, including the availability of suitable standard components, to be achieved.

The modelling system has been configured in its prototype form for the design of powertransmission systems. This has enabled a number of standard component models to be usefully used for the conceptual design of a wide variety of systems.

With this structure it is considered that additional domain knowledge, operating performance, actual life cycle performance data can readily be incorporated so that these additional considerations are considered as early as possible.

\subsection{REFERENCES}

Blessing LTM,(1994) "A Process-Based Approach to Computer-Supported Engineering Design", PhD Thesis, University of Twente, 1994.

Chakrabarti A(1991), Designing by Functions, PhD Thesis, University of Cambridge, 1991

Claypole J(1993), "Pondering on Parametrics, Professional Engineering, March 1993

Culley SJ, Corderoy MP, Ajderian NJS(1991), "The Concept of Computer Integrated Design", Proceedings of Effective CADCAM '91, IMechE, 1991.

Culley SJ, Webber SJ(1992), "Implementation Requirements for Electronic Standard Component Catalogues",_Proceedings of IMechE Journal of Engineering Manufacture, Vol.

206, pp 253-260, 1992 .

Duffey MR, Dixon JR(1990), "A Program of Research in Mechanical Design: Computer-

Based Models and Representations", Mechanisms and Machine Theory, Vol. 25, No. 3, pp383395, 1990

Engelmore R, Morgan T(1988).” Blackboard Systems” Addison Wesley Ltd., 1988

Finger S.Rinderle JR(1989), “A Transformational Approach to Mechanical Design Synthesis Using a Bond Graph Grammar" Proceedings of ASME DTM Conference Sept.1989

Finger S, Dixon JR(1989), "A Review of Research in Mechanical Design. Part I: Descriptive, Prescriptive, and Computer-Based Models of Design Processes", Research in Engineering Design, Vol. 1, pp 51-67, 1989.

Hoover SP, Rinderle JR, Finger S(1991) "Models and Abstractions in Design", Proceedings of ICED'91 Zurich, 1991

Howe A, Cohen P, Dixon JR, Simmons MK(1986), "Dominic: a Domain Independent Program for Mechanical Design", Artificial Intelligence in Engineering, Vol. 1, No. 1, 1986

Hubka V(1992). "Principles of Engineering Design", Butterworth Scientific 1992

Johnson AL, Thornton AC(1991), "Towards Real CAD", Design Studies, Vol. 12, No. 4, October 1991

Pahl G, Beitz W(1984), "Engineering Design", The Design Council, London, 1984.

Reinemuth J, Birkhofer H(1993), "Electronic Catalogue Systems for Supplied Components", Hanser Fachzeitschrift, Carl Hanser Verlag, Munchen, 1993.

Theobald G, Culley S.J. Ajderian N, Vogwell J(1993), “ The modelling of Engineering Assemblies Based on Standard Components"Proceedings of ICED'93, The Hague 1993 Ullman DG(1992a), "A Taxonomy for Mechanical Design", Research in Engineering Design, Vol. 3, 1992.

Ullman DG(1992b) " The Mechanical Design Process"_McGraw Hill Inc.1992 
Ullman DG (1993) " The Evolution of Function and Behaviour During Mechanical Design" Proceedings of ASME DTM Conference 1993

Vogwell J, Culley SJ,(1991) "A Strategy for Selecting Engineering Components", Proceedings of IMechE Journal of Engineering Manufacture, Vol. 205, pp 11-17, 1991. .Ward AC, Seering WP(1987), "An Approach to Computational Aids for Mechanical Design", Proceedings of ICED '87, Boston, USA, 1987.

.Ward AC, Seering WP(1990), "The Performance of a Mechanical Design Compiler", Computers in Engineering, 1990.

Wood TL(1994), "Fuzzy Logic Techniques Applied to the Automated Selection of Ill-Defined Standard Components", PhD Thesis, University of Bath, 1994

\subsection{BIOGRAPHY}

Steve Culley is Senior Lecturer in Engineering Design , since 1983, having previously worked in the steel, fluid power and rubber industries. He has pioneered work into the development of engineering databases of standard components, including the development of commercial packages. This work has been expanded to develop techniques to incorporate these into assemblies an sub-assemblies and also into general research on the provision of information for engineering designers.

Glyn Theobald having completed his doctorate at Bath is now Senior systems engineer with Rover Cars in the UK. 